\title{
723 WTX-613, A CONDITIONALLY ACTIVATED IFN $\alpha$ INDUKINETM MOLECULE, INDUCES ANTI-TUMOR IMMUNE RESPONSES RESULTING IN STRONG TUMOR GROWTH CONTROL IN SYNGENEIC MOUSE TUMOR MODELS
}

Ethika Tyagi ${ }^{*}$, Heather Brodkin, Josue Canales, Dan Hicklin, Nesreen Ismail, Kristin Morris, Christopher Nirschl, Andres Salmeron, Cindy Seidel-Dugan, Philipp Steiner, Zoe Steuert, Jenna Sullivan, William Winston. Werewolf Therapeutics, Cambridge, MA, United States

Background Interferon $\alpha$ (IFN $\alpha)$ was the first cytokine clinically tested as a cancer therapy. IFN $\alpha$ is a member of the type-I IFN family and activates immune responses either directly by engaging IFN $\alpha$ receptors (IFNAR) ubiquitously expressed on immune cells or indirectly by inducing chemokines that attract myeloid and lymphoid cells to the tumor site. High dose IFN $\alpha$ therapy was approved for melanoma, lymphoma, and leukemia but its use is limited by systemic toxicity and modest efficacy.

Methods WTX-613 is a novel systemically delivered IFNa2b pro-drug identified using the Predator $^{\mathrm{TM}}$ discovery platform. The inducible WTX-613 INDUKINE ${ }^{\mathrm{TM}}$ molecule is designed to deliver wild-type IFN $\alpha 2 b$ in the tumor microenvironment to reduce systemic toxicity. WTX-613 has two identical half-life extension (HLE) domains tethered to IFN $\alpha 2 b$ via a tumor protease-sensitive linker. The HLE domain supports less frequent systemic administration but importantly also prevents binding of WTX-613 to IFNAR due to steric hindrance until removal of the HLE domains by tumor proteases.

Results WTX-613 was selected as a lead molecule due to its improved in vitro profile. Since human IFNo is not functional in the mouse, a surrogate WTX-613 molecule was created consisting of mouse IFN 1 to explore anti-tumor responses in mouse syngeneic tumor models. Intraperitoneal (i.p.) administration of the WTX-613 surrogate resulted in anti-tumor responses in the more immunogenic MC38 colon model which was well tolerated by the mice. Furthermore, less immunogenic tumor models such as B16F10 melanoma and EMT6 breast carcinoma, which are generally less responsive to I/O therapy, also responded with similar anti-tumor activity. Importantly, wild-type mouse IFN $\alpha 1$ was only active in mouse models during the dosing period, and tumors grew back once treatment was stopped. However, the WTX-613 surrogate INDUKINE $^{\mathrm{TM}}$ molecule had long lasting anti-tumor activity when dosed at equimolar amounts compared to the native IFN $\alpha$ during the dosing period. The WTX-613 surrogate strongly activated $\mathrm{NK}$ and $\mathrm{CD} 8+$ cell responses and induced APC and effector cell markers in MC38 tumors. Specifically, the WTX-613 surrogate was better than native IFNa1 in inducing $\mathrm{CD} 8+, \mathrm{NK}$, and $\mathrm{DC}$ cells.

Conclusions Preclinical data obtained so far support the continued development of this innovative and differentiated engineered IFN $\alpha$ therapy and progression into clinical trials.

Ethics Approval All mouse in vivo work was performed in accordance with current regulations and standards of the U.S. Department of Agriculture and the NIH at Charles River Laboratories (Morrisville, NC and Worcester, MA).

http://dx.doi.org/10.1136/jitc-2021-SITC2021.723 